Proceedings of the

International Geometry Center

Vol. 12 , no. 2 (2019) pp. 26-42

\title{
Додатні ряди, множини підсум яких є канторвалами
}

\author{
Я. Ф. Виннишин, В. П. Маркітан, М. В. Працьовитий, \\ I. О. Савченко
}

\begin{abstract}
A set of incomplete sums (subsums) of a absolutely convergent series has been featured in studies since 1914, when a pioneer work in this direction was published by Japanese mathematician Soichi Kakey [7]. Since then, active research has begun in this direction. And already final in the direction of classification of existing "topological types" of sets of incomplete sums of absolutely convergent series are the works of $1988[4,10]$, where the following fact is proved:

If $E$ is the set of subsums of a positive term convergent series $\sum_{n=1}^{\infty} a_{n}$, then $E$ is one of the following: (i) a finite union of closed intervals; (ii) homeomorphic to the Cantor set; (iii) homeomorphic to a certain set $T$ called Cantorval, see (1.3).

Beginning in 1941, in parallel with studies of the topological properties of the sets of incomplete sums, studies of their metric properties were carried out. Metric problems (Lebesgue measure problems) are of particular relevance when the set of incomplete sums of a series is nowhere dense or is a mixture of nowhere dense sets and a union of segments.

Today, the following problems remain open in the general formulation for the set of incomplete sums of a converging positive series:

1) necessary and sufficient conditions for its nowhere density;

2) necessary and sufficient conditions for its zero-dimensionality (in the sense of Lebesgue measure);

3) its fractal properties, etc.
\end{abstract}

Колектив авторів щиро вдячний анонімному рецензентові за конструктивні зауваження та побажання, які дозволили вдосконалити дану роботу.

Ключові слова: множина неповних сум (підсум), канторвал, міра Лебега, $\varepsilon$-апроксимація лінійної множини.

DOI: http://dx.doi.org/10.15673/tmgc.v12i2.1455 
In the present paper we give a construction of a continuum family of positive series whose sets of incomplete sums are Cantorvals. Each series of this family has a property

$$
\sum_{n=1}^{\infty} a_{n}=1, \quad \varlimsup_{n \rightarrow \infty} \frac{a_{n}}{\sum_{k=1}^{\infty} a_{n+k}}=+\infty,
$$

Moreover, for any $\varepsilon>0$ there exists a series in this family with Lebesgue measure of its set of incomplete sums greater than $1-\varepsilon$.

Анотація. Наводиться конструкція континуальної сім'ї додатних рядів, множини неповних сум яких є канторвалами (об'єднанням ніде не щільної множини і множини, яка є нескінченним об'єднанням відрізків). Кожен ряд даної сім'ї має властивість

$$
\sum_{n=1}^{\infty} a_{n}=1, \quad \varlimsup_{n \rightarrow \infty} \frac{a_{n}}{\sum_{k=1}^{\infty} a_{n+k}}=+\infty,
$$

причому для будь-якого $\varepsilon>0$ в цій сім’ї існує ряд, міра Лебега множини неповних сум якого є більшою за $1-\varepsilon$.

\section{1. ВСТУП}

Розглядається збіжний додатний ряд

$$
r_{0}=\sum_{n=1}^{\infty} a_{n}=a_{1}+a_{2}+\ldots+a_{n}+r_{n}=S_{n}+r_{n}
$$

Нехай $M$ - довільна підмножина множини натуральних чисел $\mathbb{N}$. Число

$$
x(M)=\sum_{n \in M \subset \mathbb{N}} a_{n}=\sum_{n=1}^{\infty} a_{n} \varepsilon_{n}, \quad \text { де } \varepsilon_{n}= \begin{cases}1, & \text { якщо } n \in M, \\ 0, & \text { якщо } n \notin M,\end{cases}
$$

називається неповною сумою (підсумою) ряду (1.1), визначеною множиною $M$.

Множину всіх неповних сум ряду (1.1) позначатимемо через $E\left\{a_{n}\right\}$, тобто

$$
E\left\{a_{n}\right\} \equiv\left\{x \mid x=\sum_{n \in M} a_{n}, \quad M \in 2^{\mathbb{N}}\right\}
$$

Легко бачити, що множина $E\left\{a_{n}\right\}$ всіх підсум абсолютно збіжного ряду $\sum_{n \in \mathbb{N}}$ не змінюється при довільній перестановці членів ряду. Крім того, всі члени збіжного додатного ряду можна переставити у збіжну до нуля спадну (у нестрогому розумінні) послідовність. Тому при дослідженні множини $E\left\{a_{n}\right\}$ достатньо розглядати випадок монотонної спадної послідовності $\left(\left|a_{n}\right|\right)_{n=1}^{\infty}$. 
Оскільки для абсолютно збіжного ряду ряду $\sum_{n=1}^{\infty} a_{n}$ має місце рівність

$$
\sum_{n=1}^{\infty} a_{n}=S^{-}+\sum_{n=1}^{\infty}\left|a_{n}\right|
$$

де $S^{-}$- сума всіх від'ємних членів ряду, то в дослідженнях топологометричної структури множини підсум абсолютно збіжних рядів можна обмежитись розглядом додатних рядів, що ми й надалі робитимемо.

Як окремий об'єкт вивчення, множина підсум абсолютно збіжного ряду фігурує в дослідженнях з 1914 року, коли була опублікована піонерська в цьому напрямі робота японського математика Соічі Какея [7] ("Про неповні суми нескінченних рядів"), де він описав струтруру множини неповних сум, не надавши при цьому строгих доведень. В 1941 році результати Какея були перевідкриті Г. Горничем [5] та в 1948 році П. К. Меноном [9] й основний результат того часу сформульовнано у вигляді:

Теорема 1.1. Множина неповних сум $E\left\{a_{n}\right\}$ абсолютно збіюнного ряду $\sum_{n=1}^{\infty} a_{n} \in$ досконалою множиною.

1) Більш того, $E\left\{a_{n}\right\}$ є скінченним об'єднанням відрізків тоді й лише тодi, коли

$$
\left|a_{n}\right| \leqslant r_{n} \equiv\left|a_{n+1}\right|+\left|a_{n+2}\right|+\left|a_{n+3}\right|+\cdots
$$

для всіх $n$, починаючи з деякого номеру $\left(E\left\{a_{n}\right\}\right.$ е відрізком тоді й лише тодi, коли $\left|a_{n}\right| \leqslant r_{n}$ для всіх $\left.n \in \mathbb{N}\right)$.

2) Якщо ж

$$
\left|a_{n}\right|>\left|a_{n+1}\right|+\left|a_{n+2}\right|+\left|a_{n+3}\right|+\cdots
$$

для всіх достатнъо великих $n$, то $E\left\{a_{n}\right\}$ гомеоморфна класичній множині Кантора.

У згаданій роботі [7] С. Какея висунув припущення, що необхідною і достатньою умовою ніде не щільності множини $E\left\{a_{n}\right\}$ є існування зліченної кількості членів ряду, для яких $\left|a_{n}\right|>r_{n}$. Перший контрприклад до цієї гіпотези навели в 1980 р. А. Д. Вайнштейн і Б. З. Шапіро [16]. $\mathrm{y}$ роботі [11] Ф. Прус-Вішньовський, визнаючи спростування гіпотези Какея вказаними авторами, зазначає, що їх робота містить і хибні твердження. У 1984 р. Ц. Ференс [3] навів інший контрприклад для спростування гіпотези Какея, а зовсім простий приклад ряду у 1988 р. 
представили Дж. Гатрі і Дж. Німан [4]:

$$
\frac{3}{4}+\frac{2}{4}+\frac{3}{4^{2}}+\frac{2}{4^{2}}+\frac{3}{4^{3}}+\frac{2}{4^{3}}+\cdots
$$

Для цього ряду, як і для рядів із вище наведених робіт, нерівності $a_{n}>r_{n}$ і $a_{n} \leqslant r_{n}$ виконуються нескінченну кількість разів, а множина неповних сум ряду (1.2) містить відрізок $\left[\frac{2}{3}, 1\right]$, але не є скінченним об'єднанням відрізків, див. [2]. Множина неповних сум ряду (1.2) гомеоморфна множині

$$
T \equiv C \cup \bigcup_{n=1}^{\infty} G_{2 n-1}=[0,1] \backslash \bigcup_{n=1}^{\infty} G_{2 n},
$$

де $C$ - класична множина Кантора (тобто множина чисел з відрізка $[0,1]$, які записуються у трійковій системі числення з використанням двох цифр 0 та 2, або, що рівносильно - це множина неповних сум геометричного ряду $\left.\sum_{k=1}^{\infty} \frac{2}{3^{k}}\right), G_{k}-$ відкрита множина всіх чисел відрізка $[0,1]$, які мають у своєму трійковому зображенні $k$-ту цифру 1 , якщо всі попередні цифри зображення - 0 або 2.

Завершальними у напрямі класифікації існуючих "топологічних типів" множин неповних сум абсолютно збіжних рядів є роботи $[4,10]$, де доведено наступний факт.

Теорема 1.2. Множина $E\left\{a_{n}\right\}$ неповних сум збіжного додатного ряду $\sum_{n=1}^{\infty} a_{n}$ або

1) є скінченним об'єднанням відрізків, або

2) гомеоморбна множсині Кантора, або

3) гомеоморбна множині $T$.

Означення 1.3. [1, 11] Cиметричним канторвалом (або $M$-канторвалом) називається множина, яка гомеоморфна множині $T$.

Термін "канторвал" запропонували бразильські математики П. Мендес і Ф. Олівейра у роботі [8], присвяченій вивченню топологічної структури арифметичної суми двох множин канторівсъкого типу (непорожніх обмежених досконалих нуль-множин Лебега з $\mathbb{R}$ ). У цій роботі автори дали канторвалам дещо інше означення.

Означення 1.4. [8] $M$-канторвалом називається досконала підмножина числової прямої $\mathbb{R}$ така, що кожний суміжний інтервал цієї множини накопичує по обидві сторони нескінченну кількість своїх нетривіальних компонент зв'язності та суміжних інтервалів. 
Починаючи з 1941 року, паралельно з дослідженнями топологічних властивостей множин неповних сум, проводились дослідження їх метричних властивостей. Окремої актуальності метричні задачі (задачі про міру Лебега) набувають у випадку, коли множина неповних сум ряду є ніде не щільною або сумішшю ніде не щільної множини та об'єднання відрізків. Дещо пізніше коло метричних задач було розширене задачами про фрактальні властивості множин неповних сум, про їхні фрактальні розмірності (розмірність Гаусдорфа-Безиковича та інші). Першими вагомими дослідженнями в цьому напрямі були роботи Тібора Шалата [15, 14].

Незважаючи на те, що в останній час акцентовано ведуться дослідження $[1,12,6,17,18,19,20]$ властивостей множини неповних сум збіжного ряду (в основному це розгляд окремих випадків, коли члени ряду утворюють послідовність, яка володіє деякою властивістю однорідності відносно $n$ ) і на те, що за столітній період розвитку цієї теорії науковцями було отримано ряд вагомих результатів $[4,5,7,9,15,16$, $21,14]$, повний опис тополого-метричних властивостей множини $E\left\{a_{n}\right\}$ зали ється ще далеким до завершення.

Сьогодні стосовно множини неповних сум зб жного додатного ряду все ще у загальній постановці залишаються відкритими наступні проблеми:

1) про необхідні і достатні умови їі ніде не щільності;

2) про необхідні і достатні умови ㄲï нульмірності (в розумінні міри Лебега).

Ще більш складною проблемою у загальній постановці є задача про фрактальні властивості множини $E\left\{a_{n}\right\}$, хоча для деяких класів рядів це успішно зроблено, див. [15, 19, 21, 14].

Нагадаємо, що нескінченною згорткою Бернуллі, керованою числовим рядом (1.1), називається розподіл випадкової величини

$$
\xi=\xi_{1} a_{1}+\xi_{2} a_{2}+\ldots++\xi_{n} a_{n}+\ldots
$$

де $\left(\xi_{n}\right)$ - послідовність незалежних випадкових величин, які набувають значень 0 і 1.

Нескінченні згортки Бернуллі є актуальним об'єктом сучасних наукових досліджень $[21,17,19,13]$, і з ними пов'язано ряд складних проблем. Оскільки розподіл випадкової величини $\xi$ зосереджений на множині неповних сум ряду (1.1), а її спектр (мінімальний замкнений носій) або збігається з множиною неповних сум, або є ї підмножиною, то знання тополого-метричних властивостей множини неповних сум сприяють дослідженню розподілу $\xi$. 
У даній роботі ми цікавимося питанням: якої найбільшої масивності (у розумінні міри Лебега) може досягати множина неповних сум додатного монотонного ряду (1.1), для якого виконується умова

$$
\varlimsup_{n \rightarrow \infty} \frac{a_{n}}{r_{n}}=+\infty ?
$$

Наближувати множини неповних сум і геометрично інтерпретувати процес наближення можна в термінах циліндричних відрізків.

Нагадаємо, що для ряду (1.1) ииліндричним відрізком рангу $m 3$ основою $\varepsilon_{1} \varepsilon_{2} \ldots \varepsilon_{m}\left(\varepsilon_{i} \in\{0,1\}\right)$ називають відрізок $\Delta_{\varepsilon_{1} \varepsilon_{2} \ldots \varepsilon_{m}}$ з кінцями

$$
a=\sum_{i=1}^{m} \varepsilon_{i} a_{i}, \quad b=r_{m}+a .
$$

Очевидно, що $\Delta_{\varepsilon_{1} \varepsilon_{2} \ldots \varepsilon_{m} i} \subset \Delta_{\varepsilon_{1} \varepsilon_{2} \ldots \varepsilon_{m}}$ для $i=\{0,1\}$, але, взагалі кажучи,

$$
\Delta_{\varepsilon_{1} \varepsilon_{2} \ldots \varepsilon_{m}} \neq \Delta_{\varepsilon_{1} \varepsilon_{2} \ldots \varepsilon_{m} 0} \cup \Delta_{\varepsilon_{1} \varepsilon_{2} \ldots \varepsilon_{m} 1} .
$$

Легко бачити, що кінці циліндричних відрізків є точками множини $E$, а сама множина $E$ є замиканням множини всіх кінців циліндричних відрізків.

Нехай $E_{m}$ - це об'єднання всіх циліндричних відрізків рангу $m$, тобто

$$
E_{m}=\bigcup_{\varepsilon_{1}=0}^{1} \bigcup_{\varepsilon_{2}=0}^{1} \ldots \bigcup_{\varepsilon_{m}=0}^{1} \Delta_{\varepsilon_{1} \varepsilon_{2} \ldots \varepsilon_{m}} .
$$

Тоді

1) $E \subset E_{m+1} \subset E_{m}$ для всіх $m \in \mathbb{N}$,

2) $E=\lim _{m \rightarrow \infty} E_{m}=\bigcap_{m=1}^{\infty} E_{m}$.

Нехай

$$
\Delta_{\varepsilon_{1} \varepsilon_{2} \ldots \varepsilon_{m}}^{\prime} \equiv \Delta_{\varepsilon_{1} \varepsilon_{2} \ldots \varepsilon_{m}} \cap E\left\{a_{n}\right\} .
$$

Тоді, очевидно, що множина $E\left\{a_{n}\right\}$ є симетричною відносно точки $\frac{1}{2} r_{0}$, a $\Delta_{\varepsilon_{1} \varepsilon_{2} \ldots \varepsilon_{m}}^{\prime}-$ відносно середини відрізка $\Delta_{\varepsilon_{1} \varepsilon_{2} \ldots \varepsilon_{m}}$.

Не порушуючи загальності міркувань, в задачах тополого-метричного характеру можна вважати ряд нормованим, тобто з сумою, яка дорівнюе 1.

\section{2. ГолОвний оБ'ЄКТ ДОсЛІДЖЕНня}

Нехай $\left(s_{n}\right)$ і $\left(m_{n}\right)$ - послідовності натуральних чисел і $\left(a_{n}\right)$ - така послідовність додатних дійсних чисел, що 


$$
\begin{gathered}
\underbrace{a_{1}+\cdots+a_{1}}_{s_{1}+1}+\underbrace{\frac{m_{1}-1}{m_{1}} a_{1}+\cdots+\frac{m_{1}-1}{m_{1}} a_{1}}_{m_{1}}+ \\
+\underbrace{a_{2}+\cdots+a_{2}}_{s_{2}+1}+\underbrace{\frac{m_{2}-1}{m_{2}} a_{2}+\cdots+\frac{m_{2}-1}{m_{2}} a_{2}}_{m_{2}}+\ldots+\underbrace{a_{n}+\cdots+a_{n}}_{s_{n}+1}+ \\
+\underbrace{\frac{m_{n}-1}{m_{n}} a_{n}+\cdots+\frac{m_{n}-1}{m_{n}} a_{n}}_{m_{n}}+\widetilde{r}_{n}=\sum_{k=1}^{\infty} d_{k}=r_{0}=1
\end{gathered}
$$

- збіжний додатний ряд, для якого виконується умова

$$
\tilde{r}_{n}=\frac{2 a_{n}}{m_{n}}, \quad n=1,2,3, \ldots,
$$

де

$$
\tilde{r}_{n}=\sum_{k=n+1+\sum_{i=1}^{n}\left(s_{i}+m_{i}\right)}^{\infty} d_{k}=\sum_{i=n+1}^{\infty}\left(s_{i}+m_{i}\right) a_{i}
$$

a $d_{k}$ - це $k$-ий елемент послідовності утвореної з членів ряду $(2.1)$, тобто

$$
\begin{aligned}
& \underbrace{a_{1}, \ldots, a_{1}}_{s_{1}+1}, \underbrace{\frac{m_{1}-1}{m_{1}} a_{1}, \ldots, \frac{m_{1}-1}{m_{1}} a_{1}}_{m_{1}}, \\
& \underbrace{a_{2}, \ldots, a_{2}}_{s_{2}+1}, \underbrace{\frac{m_{2}-1}{m_{2}} a_{2}, \ldots, \frac{m_{2}-1}{m_{2}} a_{2}}_{m_{2}}, \ldots, \\
& \underbrace{a_{n}, \ldots, a_{n}}_{s_{n}+1}, \underbrace{\frac{m_{n}-1}{m_{n}} a_{n}, \ldots \cdot \frac{m_{n}-1}{m_{n}} a_{n}}_{m_{n}}, \ldots
\end{aligned}
$$

Зокрема,

$$
\begin{gathered}
d_{1}=\cdots=d_{s_{1}+1}=a_{1} \\
d_{s_{1}+2}=\cdots=d_{s_{1}+m_{1}+1}=\frac{m_{1}-1}{m_{1}} a_{1}, \\
d_{s_{1}+m_{1}+2}=\cdots=d_{s_{1}+m_{1}+s_{2}+2}=a_{2}, \\
d_{s_{1}+m_{1}+s_{2}+3}=\cdots=d_{s_{1}+m_{1}+s_{2}+m_{2}+2}=\frac{m_{2}-1}{m_{2}} a_{2},
\end{gathered}
$$

і т.д. Зрозуміло, що при виконанні умови (2.2) послідовності $\left(s_{n}\right)$ і $\left(m_{n}\right)$ визначають члени ряду (2.1). 
Теорема 2.1. При виконанні умов (2.1) $i$ (2.2) члени послідовності $\left(a_{n}\right)$ мають вигляд

$$
a_{n}=\frac{2^{n-1} \cdot m_{n}}{\prod_{k=1}^{n}\left(m_{k}^{2}+s_{k} m_{k}+2\right)}
$$

Доведення. Для $n=1$ маємо

$$
1=\left(s_{1}+1\right) a_{1}+\left(m_{1}-1\right) a_{1}+\widetilde{r}_{1}=\left(s_{1}+m_{1}\right) a_{1}+\frac{2 a_{1}}{m_{1}} .
$$

Звідки

$$
a_{1}=\frac{m_{1}}{m_{1}^{2}+s_{1} m_{1}+2}
$$

Враховуючи означення $\widetilde{r}_{n}$ і рівність (2.2), маємо

звідки

$$
\begin{gathered}
\tilde{r}_{n}=a_{n+1}\left(s_{n+1}+m_{n+1}\right)+\widetilde{r}_{n+1}=a_{n+1}\left(s_{n+1}+m_{n+1}\right)+\frac{2 a_{n+1}}{m_{n+1}}, \\
a_{n}=\frac{m_{n} \tilde{r}_{n}}{2}=\frac{a_{n+1} m_{n}}{2}\left(s_{n+1}+m_{n+1}+\frac{2}{m_{n+1}}\right)
\end{gathered}
$$

$$
a_{n+1}=\frac{2 m_{n+1}}{m_{n+1}^{2}+s_{n+1} m_{n+1}+2} \cdot \frac{a_{n}}{m_{n}} .
$$

Послідовно підставляючи вирази $a_{n}, a_{n-1}, \ldots, a_{1}$ з $(2.4)$, отримуємо

$$
\begin{aligned}
a_{n+1} & =\frac{2 m_{n+1}}{m_{n+1}^{2}+s_{n+1} m_{n+1}+2} \cdot \frac{a_{n}}{m_{n}}= \\
& =\frac{2 m_{n+1}}{m_{n+1}^{2}+s_{n+1} m_{n+1}+2} \cdot \frac{1}{m_{n}} \cdot \frac{2 m_{n}}{m_{n}^{2}+s_{n} m_{n}+2} \cdot \frac{a_{n-1}}{m_{n-1}}=\cdots \\
& =\frac{2^{n} \cdot m_{n+1}}{\prod_{i=1}^{n+1}\left(m_{i}^{2}+s_{i} m_{i}+2\right)} .
\end{aligned}
$$

Отже, має місце рівність (2.3).

Наслідок 2.2. Для членів та залишків ряду (2.1) мають місие наступні співвідношення:

$$
\begin{gathered}
\tilde{r}_{n}=\frac{2^{n}}{\prod_{k=1}^{n}\left(m_{k}^{2}+s_{k} m_{k}+2\right)}, \\
\frac{a_{n+1}}{a_{n}}=\frac{2 m_{n+1}}{m_{n}\left(m_{n+1}^{2}+s_{n+1} m_{n+1}+2\right)}, \quad \frac{\widetilde{r}_{n+1}}{\tilde{r}_{n}}=\frac{2}{m_{n+1}^{2}+s_{n+1} m_{n+1}+2} .
\end{gathered}
$$




\section{3. ЗАДАЧІ ТА РЕЗУЛЬТАТИ}

Нас цікавлять тополого-метричні властивості множини неповних сум ряду $(2.1)$ за умови, коли $\left(s_{n}\right)$ і $\left(m_{n}\right)$ - зростаючі послідовності натуральних чисел. Нижче ми доведемо, що множина неповних сум цього ряду містить відрізки, скориставшись простою ідеєю: якщо множина неповних сум ряду є щільною у деякому відрізку, то чей відрізок повністю належить множині неповних сум в силу замкненості останнъої. 3 цією метою ми використовуватимемо поняття в-апроксимацї множсин, яке успішно застосовувалось Цезарем Ференсом у роботі [3].

Нехай $\varepsilon-$ деяке додатне число. Будемо казати, що множина $J \subset \mathbb{R}$ $\varepsilon$-апроксимуеться множиною $U \subset \mathbb{R}$, якщо

$$
\forall x \in J \quad \exists u \in U: \quad 0 \leqslant x-u \leqslant \varepsilon .
$$

Подвійна нерівність (3.1) рівносильна такій парі нерівностей:

$$
x-\varepsilon \leqslant u \leqslant x \quad \text { i } \quad u \leqslant x \leqslant u+\varepsilon .
$$

Тому

$$
J \subset \bigcup_{u \in U}[u, u+\varepsilon]
$$

Введемо позначення:

$$
\begin{gathered}
\varepsilon_{n} \equiv \frac{a_{n}}{m_{n}}=\frac{\tilde{r}_{n}}{2}, \\
S_{n}=\sum_{k=1}^{n}\left(s_{k}+1+m_{k}\right)=n+\sum_{k=1}^{n}\left(s_{k}+m_{k}\right), \\
l_{n}=\left(m_{n}-3\right) a_{n}+\frac{2 a_{n}}{m_{n}}=\frac{a_{n}}{m_{n}}\left(m_{n}^{2}-3 m_{n}+2\right)=\frac{a_{n}}{m_{n}}\left(m_{n}-1\right)\left(m_{n}-2\right), \\
L_{n}=\left(s_{n}+3\right) a_{n}-\frac{2 a_{n}}{m_{n}}=\frac{a_{n}}{m_{n}}\left(s_{n} m_{n}+3 m_{n}-2\right), \\
D_{n} \equiv\left\{x \mid x=\sum_{i=1}^{S_{n}} \alpha_{i} d_{i}, \quad \alpha_{i} \in\{0,1\}\right\}
\end{gathered}
$$

- множина неповних сум частинної суми $d_{1}+d_{2}+\ldots+d_{S_{n}}$ ряду $(2.1)$ рангу $n$.

Лема 3.1. Множина E всіх підсум скінченного ряду

$$
\underbrace{m+m+\ldots+m}_{s+1}+\underbrace{(m-1)+(m-1)+\ldots+(m-1)}_{m}
$$


містить множину

$$
\mathbb{N} \cap[(m-1)(m-2), s m+3 m-2],
$$

де $s, m \in \mathbb{N} m a s \geqslant m-3$.

Доведення. Очевидно, що

$$
\begin{aligned}
\{0, m, 2 m,(m-2) & m, \ldots,(s+1) m\} \\
+ & \\
+\{0,(m-1) m\} & =\{0, m, \ldots,(s+m) m\} \subseteq E .
\end{aligned}
$$

Якщо $m-2 \leqslant k \leqslant s+1$ і $1 \leqslant i \leqslant m-1$, то

$$
k m+i=(k+i+1-m) m+(m-i)(m+1) \in E,
$$

а отже,

$$
[(m-2) m,(s+2) m] \cap \mathbb{N} \subseteq E .
$$

Якщо $1 \leqslant i \leqslant m-2$, то

$$
(m-2) m-i=(m-2-i) m+i(m-1) \in E,
$$

a тому,

$$
[(m-2)(m-1),(m-2) m] \cap \mathbb{N} \subseteq E
$$

Нарешті, для $1 \leqslant i \leqslant m-2$ маємо, що

$$
(s+2) m+i=(s+3+i-m) m+(m-i)(m-1) \in E,
$$

а значить $[(s+2) m, s m+3 m-2] \cap \mathbb{N} \subseteq E$.

Лема 3.2. Для довілъного $d \in D_{n}$ відрізок

$$
\left[d+l_{n+1}, d+L_{n+1}+\varepsilon_{n+1}\right]
$$

$\varepsilon_{n+1}$-апроксимуеться множиною $D_{n+1}$ за умови $s_{n} \geqslant m_{n}-3$.

Доведення. Нехай $y \in D_{n+1}$. Тоді

$$
\begin{gathered}
y=\sum_{i=1}^{S_{n+1}} \alpha_{i} d_{i}=\left(\alpha_{1} a_{1}+\alpha_{2} a_{1}+\cdots+\alpha_{s_{1}+1} a_{1}+\right. \\
\left.+\alpha_{s_{1}+2} \frac{m_{1}-1}{m_{1}} a_{1}+\cdots+\alpha_{s_{1}+m_{1}+1} \frac{m_{1}-1}{m_{1}} a_{1}\right)+\cdots+ \\
+\left(\alpha_{n+\sum_{i=1}^{\alpha}\left(s_{i}+m_{i}\right)} a_{n}+\alpha{ }_{n+1+\sum_{i=1}^{n-1}\left(s_{i}+m_{i}\right)} a_{n}+\cdots+\right. \\
\left.+\alpha s_{n+s_{n}+1+\sum_{i=1}^{n-1}\left(s_{i}+m_{i}\right)} \frac{m_{n}-1}{m_{n}} a_{n}+\cdots\right)+
\end{gathered}
$$




$$
\begin{aligned}
& +\left(\alpha_{n+1+\sum_{i=1}^{n}\left(s_{i}+m_{i}\right)} a_{n+1}+\alpha_{n+2+\sum_{i=1}^{n}\left(s_{i}+m_{i}\right)} a_{n+1}+\cdots+\right. \\
& +\alpha_{n+s_{n}+2+\sum_{i=1}^{n}\left(s_{i}+m_{i}\right)} \frac{m_{n+1}-1}{m_{n+1}} a_{n+1}+\cdots+ \\
& \left.+\alpha_{n+1+\sum_{i=1}^{n+1}\left(s_{i}+m_{i}\right)} \frac{m_{n+1}-1}{m_{n+1}} a_{n+1}\right)= \\
& =\frac{a_{1}}{m_{1}}\left(\alpha_{1} m_{1}+\alpha_{2} m_{1}+\cdots+\alpha_{s_{1}+1} m_{1}+\right. \\
& \left.+\alpha_{s_{1}+2}\left(m_{1}-1\right)+\cdots+\alpha_{s_{1}+m_{1}+1}\left(m_{1}-1\right)\right)+\cdots+
\end{aligned}
$$

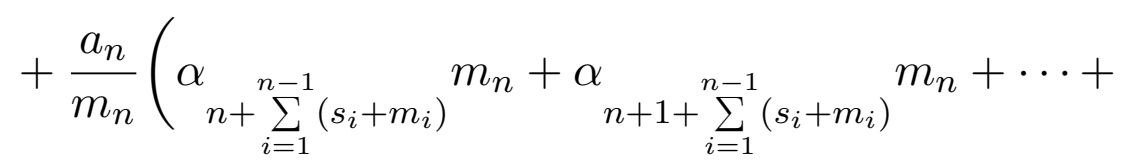

$$
\begin{aligned}
& \left.+\alpha_{n+s_{n}+1+\sum_{i=1}^{n-1}\left(s_{i}+m_{i}\right)}\left(m_{n}-1\right)+\cdots\right)+ \\
& +\frac{a_{n+1}}{m_{n+1}}\left(\alpha_{n+1+\sum_{i=1}^{n}\left(s_{i}+m_{i}\right)} m_{n+1}+\alpha_{n+2+\sum_{i=1}^{n}\left(s_{i}+m_{i}\right)} m_{n+1}+\cdots+\right. \\
& +\alpha_{n+s_{n}+2+\sum_{i=1}^{n}\left(s_{i}+m_{i}\right)}\left(m_{n+1}-1\right)+\cdots+ \\
& \left.\alpha_{n+1+\sum_{i=1}^{n+1}\left(s_{i}+m_{i}\right)}\left(m_{n+1}-1\right)\right)= \\
& =\sum_{i=1}^{n} \theta_{i} \varepsilon_{i}+\theta_{n+1} \varepsilon_{n+1}=d+\theta_{n+1} \varepsilon_{n+1},
\end{aligned}
$$

де

$$
d=\sum_{i=1}^{n} \theta_{i} \varepsilon_{i} \in D_{n} \subset D_{n+1}, \quad \varepsilon_{n}=\frac{a_{n}}{m_{n}}, \quad \theta_{n} \in A_{n},
$$

$A_{n}$ - множина всіх неповних сум скінченного ряду

$$
\underbrace{m_{n}+m_{n}+\ldots+m_{n}}_{s_{n}+1}+\underbrace{\left(m_{n}-1\right)+\left(m_{n}-1\right)+\ldots+\left(m_{n}-1\right)}_{m_{n}} .
$$

I навпаки, якщо число у має вигляд

$$
y=d+\theta_{n+1} \varepsilon_{n+1},
$$

де $\theta_{n+1} \in A_{n+1}$, то $y \in D_{n+1}$.

Оскільки, згідно з лемою 3.1, має місце 


$$
\begin{aligned}
& {\left[d+l_{n+1}, d+L_{n+1}+\varepsilon_{n+1}\right]=} \\
& =\left[d+\left(m_{n+1}\left(m_{n+1}-3\right)+2\right) \varepsilon_{n+1}, d+\left(m_{n+1}\left(m_{n+1}+3\right)-1\right) \varepsilon_{n+1}\right] \subset \\
& \subset \bigcup_{d \in D_{n+1}}\left[d ; d+\varepsilon_{n+1}\right],
\end{aligned}
$$

то згідно з (3.3), відрізок

$$
\left[d+l_{n+1} ; d+L_{n+1}+\varepsilon_{n+1}\right]
$$

$\varepsilon_{n+1}$-апроксимується множиною $D_{n+1}$. Лему доведено.

Лема 3.3. Якщо $s_{n} \geqslant 3 m_{n}$ для всіх $n \in \mathbb{N}$, то відрізок

$$
\left[\sum_{k=1}^{n} l_{k}, \frac{1}{2}\right]
$$

$\varepsilon_{n}$-апроксимуеться множиною $D_{n}$.

Доведення. Доведемо твердження за індукцією.

При $n=0$ множина $D_{0}$ складається з однієї точки 0 і згідно з лемою 3.2 , відрізок $\left[l_{1}, L_{1}+\varepsilon_{1}\right] \varepsilon_{1}$-апроксимується множиною $D_{1}$, а тому і відрізок $\left[l_{1}, \frac{1}{2}\right]$ також $\varepsilon_{1}$-апроксимується множиною $D_{1}$, оскільки

$$
\frac{1}{2} \leqslant L_{1}+\varepsilon_{1}
$$

Справді,

$$
\begin{aligned}
L_{1}+\varepsilon_{1}>L_{1} & =\frac{a_{1}}{m_{1}}\left(s_{1} m_{1}+3 m_{1}-2\right)=\frac{s_{1} m_{1}+3 m_{1}-2}{s_{1} m_{1}+m_{1}^{2}+2}= \\
& =\frac{1}{2}\left(1+\frac{m_{1}\left(s_{1}-m_{1}\right)+6\left(m_{1}-1\right)}{s_{1} m_{1}+m_{1}^{2}+2}\right)>\frac{1}{2} .
\end{aligned}
$$

Припустимо, що відрізок $\left[\sum_{k=1}^{n} l_{k}, \frac{1}{2}\right] \varepsilon_{n}$-апроксимується множиною $D_{n}$. Тоді для довільної точки $x \in\left[\sum_{k=1}^{n+1} l_{k}, \frac{1}{2}\right]$ з нерівності

$$
x \geqslant \sum_{k=1}^{n} l_{k}+l_{n+1}
$$

випливає, що

$$
x-l_{n+1} \in\left[\sum_{k=1}^{n} l_{k}, \frac{1}{2}-l_{n+1}\right] \subset\left[\sum_{k=1}^{n} l_{k}, \frac{1}{2}\right] .
$$


Згідно з припущенням і властивістю (3.3), ми можемо вказати таке $d \in D_{n}$, при якому з включення

$$
x-l_{n+1} \in\left[d, d+\varepsilon_{n}\right]
$$

випливає

$$
x \in\left[d+l_{n+1}, d+l_{n+1}+\varepsilon_{n}\right] .
$$

Для завершення доведення леми достатньо довести, що при

$$
s_{n+1} \geqslant 3 m_{n+1}
$$

має місце включення:

$$
\left[d+l_{n+1}, d+l_{n+1}+\varepsilon_{n}\right] \subset\left[d+l_{n+1}, d+L_{n+1}+\varepsilon_{n+1}\right]
$$

або, що те ж саме,

$$
l_{n+1}+\varepsilon_{n} \leqslant L_{n+1}+\varepsilon_{n+1} .
$$

Зауважимо, що згідно з наслідком 2.2

$$
\varepsilon_{n}=\varepsilon_{n+1}=\frac{\widetilde{r}_{n}}{\widetilde{r}_{n+1}}=\varepsilon_{n+1} \frac{m_{n+1}^{2}+s_{n+1} m_{n+1}+2}{2} .
$$

Тому при $s_{n+1} \geqslant 3 m_{n+1}$ маємо

$$
\begin{aligned}
L_{n+1}+\varepsilon_{n+1} & -\left(l_{n+1}+\varepsilon_{n}\right)= \\
= & \varepsilon_{n+1}\left(s_{n+1} m_{n+1}+3 m_{n+1}-1-m_{n+1}^{2}+3 m_{n+1}-\right. \\
& \left.-2-\frac{m_{n+1}^{2}+s_{n+1} m_{n+1}+2}{2}\right)= \\
= & \frac{\varepsilon_{n+1}}{2}\left(s_{n+1} m_{n+1}-3 m_{n+1}^{2}+12 m_{n+1}-8\right)= \\
= & \frac{\varepsilon_{n+1}}{2}\left(m_{n+1}\left(s_{n+1}-3 m_{n+1}\right)+12 m_{n+1}-8\right) \geqslant 0 .
\end{aligned}
$$

Згідно з лемою 3.2, відрізок

$$
\left[d+l_{n+1}, d+L_{n+1}+\varepsilon_{n+1}\right]
$$

$\varepsilon_{n+1}$-апроксимується множиною $D_{n+1}$, а тому й відрізок

$$
\left[d+l_{n+1} ; d+l_{n+1}+\varepsilon_{n}\right]
$$

$\varepsilon_{n+1}$-апроксимується множиною $D_{n+1}$. Лему доведено.

Теорема 3.4. Множина $E\left\{d_{n}\right\}$ містить відрізок

$$
\left[\sum_{k=1}^{\infty} l_{k}, \quad 1-\sum_{k=1}^{\infty} l_{k}\right]
$$


Доведення. Покажемо, що

$$
E\left\{d_{n}\right\} \supseteq\left[\sum_{n=1}^{\infty} l_{n}, \frac{1}{2}\right] .
$$

Оскільки

$$
\left[\sum_{k=1}^{\infty} l_{k}, \frac{1}{2}\right] \subseteq\left[\sum_{k=1}^{n} l_{k}, \frac{1}{2}\right]
$$

і $D_{n} \subseteq E\left\{d_{n}\right\}$ для кожного $n \in \mathbb{N}$, то з леми 3.3 випливає, що відрізок

$$
\left[\sum_{k=1}^{n} l_{k}, \frac{1}{2}\right]
$$

$\varepsilon_{n}$-апроксимується множиною $E\left\{d_{n}\right\}$ для кожного $n \in \mathbb{N}$. Зауважимо, що $\varepsilon_{n}=\frac{1}{2} \widetilde{r}_{n} \rightarrow 0$ при $n \rightarrow \infty$. Тому множина $E\left\{d_{n}\right\} \in$ щільною у відрізку $\left[\sum_{k=1}^{\infty} l_{k}, \frac{1}{2}\right]$, тобто

$$
\left[\sum_{n=1}^{\infty} l_{n}, \frac{1}{2}\right] \subseteq E\left\{d_{n}\right\}
$$

Теорему доведено.

Лема 3.5. [11] Якщо $d_{k}>r_{k}$ для деякого індексу $k$, тоді інтервал $\left(r_{k}, d_{k}\right)$ є суміюсним інтервалом до множсин неповних сум $E\left\{d_{n}\right\}$, тобто
1) $r_{k} \in E\left\{d_{n}\right\}, d_{k} \in E\left\{d_{n}\right\}$;
2) $\left(r_{k}, d_{k}\right) \cap E\left\{d_{n}\right\}=\varnothing$.

Теорема 3.6. Якщо відрізок

$$
\left[\sum_{n=1}^{\infty} l_{n}, 1-\sum_{n=1}^{\infty} l_{n}\right]
$$

непорожній, $s_{n} \geqslant 3 m_{n}$ для кожного $n \in \mathbb{N} i \varlimsup_{n \rightarrow \infty} m_{n} \geqslant 4$, то множина $E\left\{d_{n}\right\}$ е симетричним канторвалом.

Доведення. Згідно з теоремою 1.2 множина $E\left\{d_{n}\right\}$ належить до одного 3 трьох "топологічних типів". Тому для доведення того, що вона є канторвалом покажемо, що $E\left\{d_{n}\right\}$ не належить до двох інших. 
Множина $E\left\{d_{n}\right\}$ не є гомеоморфною множині Кантора, оскільки згідно з теоремою 3.4 містить відрізок

$$
\left[\sum_{k=1}^{\infty} l_{k}, 1-\sum_{k=1}^{\infty} l_{k}\right]
$$

Покажемо, що $E\left\{d_{n}\right\}$ не є скінченним об'єднанням відрізків. Припустимо супротивне: $E\left\{d_{n}\right\}$ є скінченним об'єднанням відрізків, тобто

$$
E\left\{d_{n}\right\}=\left[0, c_{1}\right] \cup\left[c_{2}, c_{3}\right] \cup \ldots \cup\left[c_{n}, 1\right]
$$

де

$$
0<c_{1} \leqslant c_{2} \leqslant c_{3} \leqslant \ldots \leqslant c_{n}<1 .
$$

Виберемо номер $k$ так, що $a_{k}<c_{1}$ i $m_{k} \geqslant 4$. Тоді

$$
0<\widetilde{r}_{k}=\frac{2 a_{k}}{m_{k}}<\frac{m_{k}-1}{m_{k}} a_{k}<c_{1} .
$$

Оскільки, згідно з лемою 3.5 інтервал $\left(\widetilde{r}_{k}, a_{k}\right)$ буде суміжним інтервалом до множини $E\left\{d_{n}\right\}$, то з цього випливає, що множина неповних сум $E\left\{d_{n}\right\}$ не містить відрізок $\left[0, c_{1}\right]$, що суперечить нашому припущенню. Тому $E\left\{d_{n}\right\}$ не є скінченним об'єднанням відрізків.

Отже, множина $E\left\{d_{n}\right\}$ є симетричним канторвалом.

Теорема 3.7. Для довільного $\varepsilon>0$ існують послідовності $\left(s_{n}\right),\left(m_{n}\right)$, $i\left(a_{n}\right)$ такі, що виконуються наступні умови:

(1) $\sum_{n=1}^{\infty} d_{n}=1$

(2) $\tilde{r}_{n}=\frac{2 a_{n}}{m_{n}}$ для кожного $n \in \mathbb{N}$, де $\widetilde{r}_{n}=\sum_{k>n}\left(s_{k}+m_{k}\right) a_{k}$;

(3) $\varlimsup_{n \rightarrow \infty} \frac{d_{n}}{\sum_{n=1}^{\infty} d_{n}}=\infty$;

(4) множина $E\left\{d_{n}\right\}$ е канторвалом, міра Лебега якого є більшою за $1-\varepsilon$.

Доведення. Візьмемо довільну строго зростаючу послідовність $\left(m_{n}\right)$ і виберемо послідовність $\left(s_{n}\right)$ таку, що

$$
s_{n} \geqslant 3 m_{n} \text { i } \frac{\left(m_{n}-1\right)\left(m_{n}-2\right)}{m_{n}\left(s_{n}+m_{n}\right)} \leqslant \frac{\varepsilon}{2}
$$

для кожного $n \in \mathbb{N}$. Тепер для кожного $n \in \mathbb{N}$ маємо

$$
l_{n}=\frac{a_{n}}{m_{n}}\left(m_{n}-1\right)\left(m_{n}-2\right)=a_{n}\left(s_{n}+m_{n}\right) \frac{\left(m_{n}-1\right)\left(m_{n}-2\right)}{m_{n}\left(s_{n}+m_{n}\right)} \leqslant \frac{\varepsilon}{2}\left(s_{n}+m_{n}\right) .
$$


Toмy

$$
\sum_{n=1}^{\infty} l_{n} \leqslant \sum_{n=1}^{\infty} \frac{\varepsilon}{2}\left(s_{n}+m_{n}\right)=\frac{\varepsilon}{2} \sum_{n=1}^{\infty}\left(s_{n}+m_{n}\right)=\frac{\varepsilon}{2} \sum_{k=1}^{\infty} d_{k}=\frac{\varepsilon}{2} .
$$

Отже,

$$
\lambda\left(E\left\{a_{n}\right\}\right)>\lambda\left(\left[\sum_{n=1}^{\infty} l_{n}, 1-\sum_{n=1}^{\infty} l_{n}\right]\right)=1-2 \sum_{n=1}^{\infty} l_{n} \geqslant 1-\varepsilon .
$$

Теорему доведено.

\section{ЛІтерАТУРА}

[1] T. Banakh, A. Bartoszewicz, S. Glab, E. Szymonik. Algebraic and topological properties of some sets in $\ell_{1}$. Colloq. Math., 129(1):75-85, 2012, doi: $10.4064 / \mathrm{cm} 129-1-5$.

[2] Wojciech Bielas, Szymon Plewik, Marta Walczyńska. On the center of distances. Eur. J. Math., 4(2):687-698, 2018, doi: 10.1007/s40879-017-0199-4.

[3] C. Ferens. On the range of purely atomic probability measures. Studia Math., $77(3): 261-263,1984$, doi: 10.4064/sm-77-3-261-263.

[4] J. Guthrie, J. Nymann. The topological structure of the set of subsums of an infinite series. Colloq. Math., 55(2):323-327, 1988, doi: 10.4064/cm-55-2-323-327.

[5] H. Hornich. Über beliebige Teilsummen absolut konvergenter Reihen. Monatsh. Math. Phys., 49:316-320, 1941, doi: 10.1007/BF01707309.

[6] Rafe Jones. Achievement sets of sequences. Amer. Math. Monthly, 118(6):508-521, 2011, doi: 10.4169/amer.math.monthly.118.06.508.

[7] S. Kakeya. On the set of partial sums of an infinite series. Tôhoku Sci Rep., (4):159-163, 1914, doi: 10.11429/ptmps1907.7.14_250.

[8] P. Mendes, F. Oliveira. On the topological structure of the arithmetic sum of two cantor sets. Nonlinearity, 7(2):329-343, 1994, doi: 10.1088/0951-7715/7/2/002.

[9] P. Menon. On a class of perfect sets. Bull. Amer. Math. Soc., 54:706-711, 1948, doi: 10.1090/S0002-9904-1948-09060-7.

[10] J. Nymann, R. Sáenz. On a paper of Guthrie and Nymann on subsums of infinite series. Colloq. Math., 83(1):1-4, 2000, doi: 10.4064/cm-83-1-1-4.

[11] F. Prus-Wiśniowski. Beyond the sets of subsums.

[12] B. Solomyak. On the random series $\sum \pm \lambda^{n}$ (an Erdos problem). Ann. of Math. (2), 142(3):611-625, 1995, doi: 10.2307/2118556.

[13] P. Varjú. Recent progress on Bernoulli convolutions. In European Congress of Mathematics, pages 847-867. Eur. Math. Soc., Zürich, 2018, doi: 10.17863/CAM. 17352.

[14] T. Šalát. Hausdorff measure of linear sets. Czechoslovak Math. J., 11 (86):24-56, 1961.

[15] Tibor Šalát. Absolut konvergente Reihen und das Hausdorffsche Mass. Czechoslovak Math. J., 9(84):372-389, 1959.

[16] А. Д. Вайнштейн, Б. З. Шапиро. О строении множества $\alpha$-представимых чисел. Изв. вузов. Матем., (5):8-11, 1980.

[17] Я. В. Гончаренко. Згортки розподілів сум випадкових рядів спеціального виду. Нaукові записки НПУ імені М.П. Драгоманова. Фізико-математичні науки, (4):216$232,2003$. 
[18] Я. В. Гончаренко, М. В. Працьовитий, Г. М. Торбін. Тополого-метричні і фрактальні властивості множини неповних сум знакододатного ряду та розподілів на ній. Науковий часопис НПУ імені М.П. Драгоманова. Фізико-математичні науки, (6):210-224, 2005.

[19] Я. В. Гончаренко, М. В. Працьовитий, Г. М. Торбін. Фрактальні властивості деяких згорток Бернуллі. Теор. ймовірност. матем. статист., 79:34-49, 2008.

[20] Н. О. Корсунь, М. В. Працьовитий. Про множину неповних сум знакододатних рядів з однією умовою однорідності та узагальнення двійкового зображення чисел. Науковий часопис НПУ імені М.П. Драгоманова. Фізико-математичні науки, (10):28-39, 2009.

[21] М. В. Працьовитий. Фрактальний підхід у досліджсеннях сингулярних розподілів. Київ: Вид-во НПУ імені М. П. Драгоманова, 1998.

\section{Я. Ф. Виннишин}

ПРИРОДНИЧО-НАУКОВИЙ ЛІЦЕЙ №145 М. КИЄВА

Email: ucrcon@bigmir.net

В. П. Маркітан

НАЦІОНАЛЬНИЙ ПЕДАГОГІЧНИЙ УНІВЕРСИТЕТ ІМЕНІ М.П. ДРАГОМАНОВА

Email: v.p.markitan@npu.edu.ua

М. В. Працьовитий

ІНСТИТУТ МАТЕМАТИКИ НАН УКРАЇНИ, НАЦІОНАЛЬНИЙ ПЕДАГОГІЧНИЙ УНІВЕРСИТЕТ IMEНI М.П. ДРАГОМАНОВА

Email: prats4444@gmail.com

\section{I. О. Савченко}

НАЦІОНАЛЬНИЙ ПЕДАГОГІЧНИЙ УНІВЕРСИТЕТ ІМЕНІ М.П. ДРАГОМАНОВА

Email: igorsav4enko@ukr.net 